\title{
Cognition, Communication, and Co-operation in Living Systems. Biosemiosis in the Context of Self-organisation
}

\author{
Wolfgang Hofkirchner ${ }^{1,2}$, Günther Ellersdorfer ${ }^{2}$
}

\author{
${ }^{1}$ ICT\&S Center \\ University of Salzburg \\ Sigmund-Haffner-Gasse 18, 5020 Salzburg, Austria \\ 2Unified Theory of Information Research Group \\ c/ o Institute of Design and Technology Assessment \\ Vienna University of Technology \\ Favoritenstraße 9, 1040 Vienna, Austria \\ wolfgang.hofkirchner@sbg.ac.at
}

\begin{abstract}
In the perspective of an as yet-to-be-developed Unified Theory of Information as part of an as yet-to-bedeveloped theory of evolutionary systems semiosis plausibly coincides with self-organisation.

A concept of sign processes that is flexible enough to perform two functions as follows is everything that is required for this framework. It must relate to the most various manifestations of sign processes, thus enabling a variety of scientific disciplines to use a common concept where it seems appropriate; at the same time, it must be precise enough to fit the unique requirements of any individual branch of science dealing with a
\end{abstract}

concrete manifestation. Different types of sign processes have to be related to, if not derived from (albeit in a nonformal way), different types of self-organisation.

The paper illustrates how the relationship between the genus proximum of semiosis in self-organising systems and the differentia specifica of semiosis in biota may be approached.

Keywords: Autopoiesis, functions, subject-object-dialectic

\section{Introduction}

Starting point of our deliberations is a bundle of basic assumptions specified in the following list:

The concept of information (generation) as defined in terms of self-organising systems by the Unified Theory of Information (e.g. Ellersdorfer and Hofkirchner 1994, Fenzl et al. 1998, Hofkirchner and Stockinger 2003) and the concept of sign (generation) as defined in Peirceian triadic semiotics can be made co-extensive. A sign in the wider sense of the term may be defined as a relation between a sign in the narrower sense of the term and an object that is relative to somebody or something that by producing this relation creates meaning. This formulation of sign processes can easily be reinterpreted in terms of self-organising systems. The somebody or something equals a self-organising system, the object equals a perturbation of the self-organising system, and the sign in the narrow sense equals the order the selforganising system is building up in respondence to the perturbation. Then information is the relation between the self-organised order and the perturbation and this relation is produced by the self-organising system and hence is relative to it and meaningful to it. Semiosis as sign process is tantamount to information as a process.

Semiotics deals with sign processes or information processes. The units of semiosis or information processes are self-organising systems. The units of biosemiosis are living systems.

Living systems represent a special type of self-organising systems. They are characterised by a special quality that makes them distinct from other forms of self-organising systems.

Living systems manifest subjectivity. But it is not this feature that makes them distinct from other selforganising systems. Each self-organising system manifests subjectivity albeit to an extent that depends on the stage of evolution the system is located in. The minimal unit of subjectivity is a something that is provided with a minimal quantum of degrees of freedom to act. This something is the most rudimentary and most primitive subject. 


\section{Subjects and Objects}

Speaking of subjectivity means returning to the terms subject and object. According to our point of view, the difference between subject and object may be seen in that a subject is capable of determining itself while an object is not. An object is something that is determined by something that is not itself. Being a subject supersedes being merely an object. While an object has no possibility of acting in ways different from merely reacting to external determinants, a subject is capable of responding in its own, unequivocal way, that is, it can make use of degrees of freedom, of freedom of choice, of choice between options all of which it disposes of and thus makes the internal determine.

\subsection{The Dialectic between Subject and Object and the Nature of Information}

Subjects and objects are related. The concepts are correlative. This has to be clarified in order to understand the nature of information or semiosis. This, in turn, can only be achieved by a proper understanding of how a subject joins up with an object. We envisage the dialectic between subject and object in the following way:

On the one hand, there is something that makes another subject to it. What effects the subjection is the subject. What is being subjected is the object. On the other hand, the object objects to its subjection and the subject is confronted by an objection. The fundamental relationship of subject and object is the constant conflict between these two opposing tendencies.

The dialectical nature of this relationship is revealed by the following:

- firstly, the sides are opposed to each other, that is, the subject is the opposite of the object and vice versa;

- secondly, they presuppose each other, that is, without a subject there is no object and vice versa;

- thirdly, they are asymmetrical, that is, subject and object play different roles when opposing each other (the subject subjects, the object objects).

As a dialectical relationship it tends toward spiralling up. Spiralling up occurs when the subject, impressed by the objection, sets out to subject the object in a different way. Then subject $\mathrm{S}$ evolves into a modified, restructured subject that disposes of a form $\mathrm{F}$, which represents another quality.

Now, if subject $S$ does so, - if it develops form $F$ in respect to its object $O$, which objects in a certain way to being subjected in a certain way, - then we can say that the object $O$ gives rise to a new form $F$ of the subject $S$ or the subject itself gives rise to a new form of itself with regards to the object. We can say the object "in-forms" the subject or the subject "in-forms" itself on behalf of the object - to "in-form" in the sense of to give rise to a new form. The result of this process may be called "in-formation", which is the new form $F$ related to object $O$ by the activity of $S$ or the relation of $F$ to $O$ by $S-S(F, O)$ - while keeping in mind that this relation is relative to $S$ and cannot be reduced to a dyadic relation of $F$ and $O$. It is a kind of meta-relation, or a triad.

Thus, information is generated by subjects in relation to objects. Each change on the side of the subject in subject-object-relationships is tantamount to the generation of information.

This makes clear why information is both subjective and objective. It refers both to a subject and an object because it is the subject that is being informed and the object about which information is simultaneously.

Information, finally, does not exist outside of subject-object-relationships, because the informing process needs subjects to be informed and objects to be informed about. 


\subsection{The Rise of Subjectivity and Information Generacy}

The making of something subject to oneself which makes oneself a subject undergoes a process of unfolding so as to let us distinguish between different types of subjects according to the degree of subjectivity they manifest (Hofkirchner et al. forthcoming).

The minimal unit of subjectivity is a something that is provided with a minimal quantum of degrees of freedom to act. This something is the most rudimentary and most primitive subject. It differs fundamentally from being an object, that is, something that does not dispose of any option to act.

An object which has no option available strictly acts according to the Aristotelian causa efficiens and causa materialis, while a subject's act does include causa finalis and causa formalis as well, for there is some end toward which the subject directs its action and there is some form which the subject implements through acting. End and form are options at the disposal of the subject. They are selected Out of a plural of options which make up the degrees of freedom.

According to different types of subjects there are different types of information generated by the subjects. The more degrees of freedom, the more sophisticated the information generation is. To draw a distinction is to create information. To choose an option is to draw a distinction. The more options there are, the higher-developed the creation of information is.

\subsection{Intrasubjective, Intersubjective and Supra-Subjective Information Generation}

There are different cases of the relationship between a subject and its object corresponding to the nature of the object:

- the object may be a simple one,

- it may be a co-subject,

- it may be a super-subject.

A simple object is an object which is no subject at all or it is a subject of a simpler kind than that of the subject in question; a co-subject is a subject of the same kind; and a super-subject is a subject of a more complex kind (usually one composed of co-subjects).

In the first case, the opposing tendencies are subjection and objection tending toward spiralling up in a three-step process:

- the subject acts on the object (subjection),

- the object reacts (objection),

- the subject changes its action by taking into account the reaction of the object to its past action (new subjection).

Regarding information or sign generation, these steps involve assimiliation - non-affordance accommodation (assimilation and accommodation being terms introduced by J. Piaget $(1976,1980)$ and affordance a term coined by J. J. Gibson $(1950,1966,1979)$ ). Assimilation is the informational or semiosic aspect of subjection. Affordance means the degree to which the object affords being subjected, nonaffordance the degree to which it does not. Finally, accommodation is what happens informationally or semiosically if the subject adapts to the object. Accommodation takes precedence over the next round of trying to subject the object. Thus intrasubjective information, that is, information in a cognitive sense is created.

In the second case, not only cognition is involved. A number of (at least two) (co-)subjects interact. The opposing tendencies can be called countering intersubjectification processes showing the following steps:

- subject $A$ acts on a subject $B$ (intersubjectification by $A$ ),

- subject $B$ reacts (intersubjectification by B), 
- and subject $A$ changes its action by taking into account the reaction of subject $B$ to its past action (new intersubjectification by $A$ ).

In informational or semiotic terms, in both subject $A$ and subject $B$ information generation and sign production is involved. Subject $A$ bases its interaction with the other subject upon its being informed by this very subject, and so does subject $B$. That is, subject $A$ starts the process with a given information. It reaches out to subject $B$. And subject $B$ informs itself about subject $A$ 's reaching out. This process can then be continued by subject $B$. Thus the intersubjective information generation involves internal information generation with each of the sides. This ties up with the information-message-understanding distinction introduced by N. Luhmann (e.g. 2001). Information in a communicative sense is created.

In the third case, not only communication takes place. A quorum number of subjects co-act and the outcome of this very co-action is a super-subject which, in turn, constrains and enables the subjects. The opposing tendencies may be called objectification and subjectification. The three steps of the spiralling-up process are as follows:

- subjects $A$ and $B$ and $C$... act conjointly on a super-subject (objectification),

- the super-subject reacts (subjectification),

- subject $A$ or subject $B$ or subject $C$... changes its contribution to the joint action by taking into account the reaction of the super-subject to the past action of $\mathrm{A}$ or $\mathrm{B}$ or $\mathrm{C} \ldots$ (new objectification).

In the perspective of information generation and sign production, by communicating with each other subjects produce supra-subjective information which informs them, in turn. This is in line with the Luhmannian categories of variation-selection-stabilisation (ibd.). Information in a co-operative sense is created.

\section{Living Systems}

It is important to notice that evolving, that is, self-organising systems play the part of subjects and objects as well.

This new outlook provides a framework within which the coincidence of self-organisation and information is plausible. Information is then every process (and every outcome of a process) where the system changes its behaviour, its state, or its structure due to some given conditions (Fenzl et al. 1996). Insofar as in each self-organising process the activity that is performed by the system in its own way is nevertheless triggered by something that is not the system itself, each self-organising process establishes informational relations, i.e., relations between the triggers, the system itself, and its activities (including the products of these activities).

What we get here is the equation self-organisation = information. Where we started from is the equation sign $=$ information. Thus, the overall result is the equation sign $=$ selforganisation.

Different manifestations of sign processes, then, will have to be linked to different manifestations of self-organising systems.

\subsection{Living Systems' Information Generacy}

To look for a general theory of evolutionary systems means to stress that being a self-organising system is the genus proximum of the whole bundle of different self-organising systems while acknowledging that there have to be markers that demarcate the differentiae specificae of ever more complex self-organising systems.

On the basis of the well-known differentiation between the physical-chemical, the biotic and the social (human, cultural) realms, and on the basis of the many findings that imply that in these realms there do 
exist self-organising processes, we have to devise a theory that logically includes the type of selforganisation that is found at a later stage of evolution as instance of a universal that is made up of just that type and the preceding type, namely, social self-organisation as a concretisation of biotic self-organisation (which has two parts: social biotic self-organisation and simple, nonsocial biotic self-organisation) and the biotic one as concretisation of the physical-chemical one (which again has two parts: biotic physicalchemical self-organisation and simple, nonbiotic physical-chemical self-organisation).

Living systems share with non-living self-organising ones the physical-chemical aspect of selforganisation: the dissipation of energy of higher entropy than that of the imported energy.

Living systems transform nutrients, obtained from the environment in most cases to ATP (adenosine triphosphate), the basic universal currency of free energy $\left(\Delta \mathrm{G}^{\circ}=\right.$ the standard free energy at $\left.\mathrm{pH} 7\right)$ in all living systems. This free energy is needed e.g. in the performance of mechanical work in muscle contractions and other cellular movements, the active transport of molecules and ions, and the synthesis of macromolecules and other biomolecules from simple precursors (Stryer 1988). The free energy used in these processes, which maintain an organism in a state that is far from equilibrium, is derived from the environment in that way, that phototrophic organisms obtain it by trapping light energy and chemotrophic organisms obtain this energy by the break-down of foodstuffs. Before this free energy can be used it is transformed into a special form, to be used for motion, active transport and biosynthesis. In most processes, this special carrier of free energy is the molecule adenosine tri-phosphate (ATP).

Energy is stored in the covalent bonds between phosphates, with the greatest amount of energy (approximately $7 \mathrm{kcal} / \mathrm{mole}$ ) in the bond between the second and third phosphate groups. This covalent bond is known as a pyrophosphate bond. This amount of energy is released, when the covalent bond is cleaved.

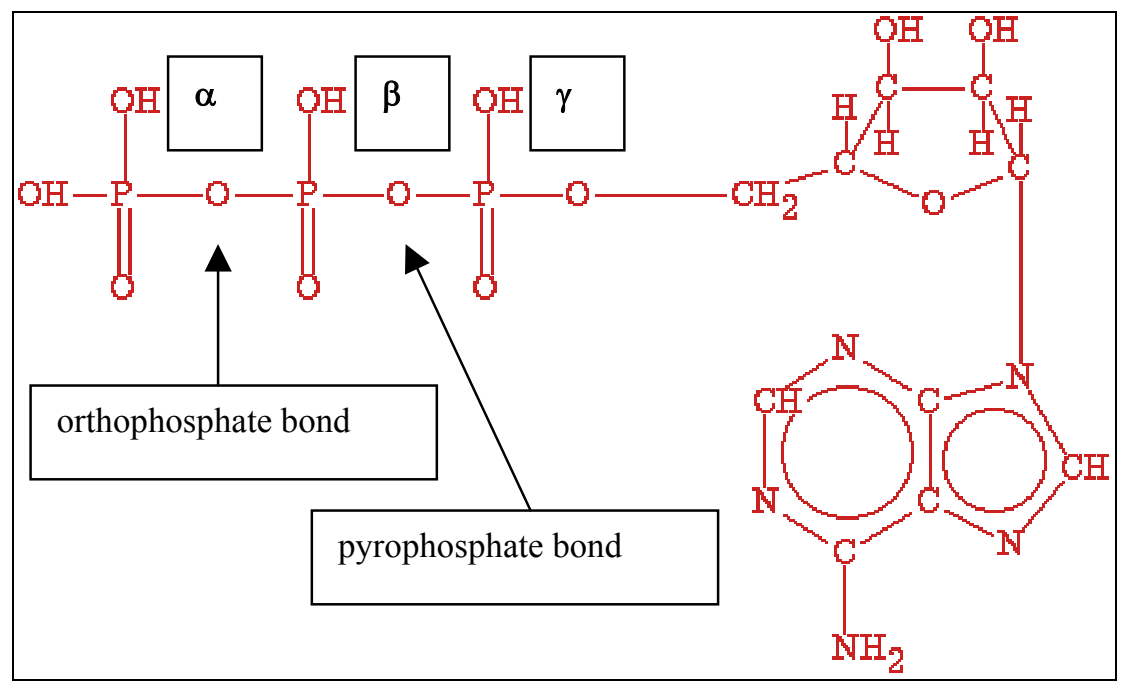

Figure 1: The chemical structure of the most important energy providing molecule in living systems: the adenosine tri-phosphate (ATP)

In ATP the energy is stored in the covalent bonds between phosphates, where the greatest amount of energy is captured in the covalent bond between the $\beta$ and $\gamma$ phosphate group, which is known as the pyrophosphate bond. An energy amount of approximately $-7 \mathrm{kcal} / \mathrm{mol}$ is released, when this covalent bond is cleaved and gets freely available as fuel molecule for metabolic activities within the cell.

Thus free energy is liberated when ATP is hydrolyzed to adenosine di-phosphate (ADP) and orthophosphate $\left(\mathrm{P}_{\mathrm{i}}\right)$ [1] or when ATP is hydrolyzed to adenosine mono-phosphate (AMP) and pyrophosphate $\left(\mathrm{PP}_{\mathrm{i}}\right)$ [2]. The chemical reaction for the hydrolytic break down and vice versa formation of ATP from ADP and orthophosphate is a bi-directional chemical reaction and can be written as: 
[1]

[2]

$$
\begin{array}{lll}
\text { ATP }+\mathrm{H}_{2} \mathrm{O} & \leftrightarrow & \text { ADP }+\mathrm{P}_{\mathrm{i}}+\mathrm{H}^{+} \\
\text {ATP }+\mathrm{H}_{2} \mathrm{O} & \leftrightarrow & \text { AMP }+\mathrm{PP}_{\mathrm{i}}+\mathrm{H}^{+}
\end{array}
$$$$
\Delta \mathrm{G}^{\circ}=-7,3 \mathrm{kcal} / \mathrm{mol}
$$

$\Delta \mathrm{G}^{\circ}=-7,3 \mathrm{kcal} / \mathrm{mol}$

The free energy $\Delta G^{\circ}$ liberated in the hydrolysis of ATP is harnessed to drive reactions that require an input of free energy as described above.

ATP, AMP and ADP are inter-convertible by the action of a specific enzyme (adenylate kinase), and the reaction [3] can be written as:

$$
A T P+A M P \quad \leftrightarrow \quad A D P+A D P
$$

A third conformational variant of the molecule ATP (besides ADP and AMP) is the cyclic form of its mono-phosphate cAMP, which can also be converted by the specific action of an enzyme (adenylate cyclase), and the reaction [4] can be written as:

$$
\text { ATP } \quad \rightarrow \quad \text { cyclic AMP + PP }
$$

ATP molecules are general energy molecules involved in all major metabolic circuits within any type of cells. As an example it should be mentioned that from one molecule of glucose, which is transformed into free available energy in the Krebs-Cycle, 2 molecules of ATP are generated. This ATP is the basic energy supplier in any anabolic process in the cell such as biosynthesis of proteins and enzymes as well as structural components to establish the cells integrity. In higher organisms we also see ATP as an important component in the transmission of neuronal signal e.g.: in the vertebrate neuronal system, also as one of the four nucleotides (ATP, GTP, CTP, TTP) it is a component of the genetic information polymers, the constituents of DNA and RNA. Also the cyclic form of adenosine mono-phosphate (cAMP) is involved in a variety of biochemical reactions within the cell, which acts as a controlling molecule in many biological processes, where a signal coming to the surface of the cell is mediated by cAMP towards inside the cell, a so called second messenger. Finally, as we will discuss later cAMP acts as a signalling molecule between different individual cells e.g. in the slime mould Dictyostelium discoideum, or the fruiting myxobacterium Stigmatella aurantiaca.

So we can summarize that this phosphorylated adenosine in its different inter-convertible forms as shown above acts as powerful and ubiquitous agent in different functions and on different level of complexity in living systems:

1. As energetic agent:

A) The energy currency at different levels of the metabolism and specially the anabolism

2. As informational agent:

A) As structural component within the polymeres of the genetic information, the DNA and the RNA

B) As second messenger mediating external information within the cellular context of a living system (cognition)

C) As neurotransmitter in synaptic gaps, thus routing neuronal events within an organismic context (communication)

D) As signalling molecule between individual cells of the same species, as response to environmental changes and trigger of concerted activities (co-operation)

In comparison to more basic systems e.g.: the Benárd cell, where a steady flow of energy in form of heat enables the formation of the typical "cells" we postulate the steady flow of ATP in the living cell as the basic activity for self-organisation activity in the living cell. The self generation of this basic energy currency contributes to the break-down and build-up within all other metabolic activities in the cell. The 
main difference to the mere physical system as mentioned in the Benárd cell system to the living cell system is, that the maintenance of the energy flow is an intrinsic function of the living cell by itself, contributing to the autopoietic property of the living cell, in the sense, as mentioned by Humberto Maturana and Francisco Varela in their theoretical concept (1992).

\begin{tabular}{|c|l|l|l|}
\hline Type of energy & Source & System & Mechanism \\
\hline $\begin{array}{c}\text { Caloric energy (heat) } \\
\begin{array}{c}\text { Chemical energy } \\
\text { (catalysis) }\end{array}\end{array}$ & Extrinsic & Benárd Cell & Dissipation \\
\hline $\begin{array}{c}\text { Metabolic energy } \\
\text { (ATP) }\end{array}$ & Intrinsic & $\begin{array}{c}\text { Belousov-Zhabotinski } \\
\text { Cell }\end{array}$ & Dissipation \\
\hline
\end{tabular}

Table 1: Comparison of different self-organising systems in respect of their energy source

By converting all energy into this ATP-molecule the cell can use it for diverse metabolic activities. This condition enables the cell to do self-organising processes on a more complex level which results in the concerted action of diverse intracellular cycles. Thus by establishing such structurally based cycles also functionally new qualities emerge within the cell.

The intracellular generation of ATP is a process typical for all living systems and serves as the basic energy currency, which is the driving force for all metabolic activities inside the cell. From our point of view ATP is the agent who mediates the establishment of the conditions necessary for the continuous throughput of energy, which is constituent for all open dynamic systems, here in this context of living, autopoietic systems. The ability to generate and appropriate this intrinsic energy source gave rise to living systems.

Assuming a defined quality of energy available within a living system it should be possible to set the boundary conditions to trigger self-organized processes within the living system without destroying the system identity, but contribute to a more advanced engineering of new properties of the system. Intrinsic generated ATP fulfills such prerequisites and can thus be designated as an autopoietic source of "high quality energy" that maintains the self-organisation of the living system. Taking these features of ATP into account we can look at this molecule as a free available and auto-generated driving force of selforganising processes in the living cellular and organismic system.

In analogy to the constant input of thermic energy necessary for the emergence of Benárd cells (dissipative self-organising systems in the physical realm) ATP-energy is also constant available in autopoietic self-organising systems that belong to the biological realm, that enables a multiplicity of emergent phenomena, which contribute to the organismic (process-based) status of a living cell.

So we can say that the differentia specifica of living systems in respect to their genus proximum, the physical-chemical systems, is based on the ability to exploit this "high quality energy" component as provided by ATP and its inter-convertible variants ADP, AMP and cAMP.

All emergent phenomena depend on a continuous energy-flow through the open system. As shown by Prigogine (e.g. 1980) is the dissipation of free energy within an open dynamic system the driving force of self-organisation in physical and chemical systems. To assume that this force is also driving selforganisation in living systems is only half the truth. For sure there are such processes in the living system (e.g.: $\mathrm{Ca}^{2+}$-cascades in nerve-impulse transmission, heart beat maintenance, micelle formation, selfassembly of virus capsides etc.), but the majority of processes constituting the functionality of a living cell depend on the "high quality energy" of the auto-generated ATP. 
Looking at autopoietic systems as basically ATP-driven should enable the design of experimental setups with the aim to detect the cellular ATP level with respect to complex reaction pathways and hypercycles. On the other hand should the controlled manipulation of intracellular ATP levels reveal new insights about the organisational aspects of biochemical reaction pathways. Tuning of the ATP level combined with the monitoring of specific reaction products, should enable to define values for specific emergent properties observable as macroscopic phenomena, such as pattern formation.

That is, by displaying the ability to maintain the far-from-equilibrium state through stock-piling energy in form of ATP biotic systems distinguish themselves from nonbiotic simple physical-chemical self-organising systems. Thus, the differentia specifica of living systems which according to the degree to which they unfold subjectivity is just another degree of freedom they enjoy, can - in terms of evolutionary system theory - be characterised by another phase of self-organisation they are able to undergo, or by another level of self-organisation they display. While simple dissipative systems show one phase or level, do autopoietic ones show two. It is the implicit end that makes them have a two-phased metamorphosis and the resilient form that makes them have a two-levelled architecture.

And accordingly, the type of information generation is specified. Due to the second phase or level they have in comparison with simple dissipative systems, autopoietic systems generate also two distinct categories of information. What is the pattern as outcome of information generation in simple dissipative systems turns into a structure that gets a function in autopoietic systems. The structure represents the outcome of the first self-organisation cycle, the function the outcome of the second one. Structure and function are related to each other.

\subsection{Living Systems' Internal, Interfacial and External Information Generacy}

The information generation in living systems manifests itself in different dimensions which are due to different phases in metasystem transitions or different levels in supersystem hierarchies recalling the metasystem transition.

- According to the intrasubjective generation of information, bio-cognition is internal to living systems.

- According to the intersubjective generation of information, bio-communication is interfacial between living systems.

- And according to the supra-subjective generation of information, bio-co-operation is external to living systems.

Internal information generation is a process that is the only one that takes place in the initial phase of a metasystem transition or at the bottom level of a supersystem. Interfacial information generation is a process that is added in the intermediate phase of a metasystem transition or at the intermediate level of a supersystem. External information generation is a process that is added in the integral phase of a metasystem transition or at the upper level of a supersystem.

In the perspective of a theory of evolutionary systems, bio-cognition as internal to a living system is made up of two grand cycles of self-organisation.

- The structure that is organised by a living system is what is known as sensation.

- The function that is attributed to the sensation by the living system is known as motivation.

Both together comprise the living system's ability to cognise which can be termed "psyche".

Bio-communication which is located at the interface of biosystems is made up of another two cycles,

- "re-presentation" being the first one,

- reorientation the second one.

Re-presentations represent the structure, while reorientation represents the function of that structure. By means of both biosystems achieve anticipations. This communicability of biosystems may be called "signalability". 
Bio-co-operation, finally, concerns the division and bringing together of functions between biosystems.

- On the one hand, there are specialisations,

- but on the other, the special structures have the function to complement each other for a common whole.

This may be called "organicity", since it makes up the basic feature of organisms and organic order.

The slime mould Dictyostelium discoideum is an eukaryotic micro-organism that occurs in forest soils spread all over the world in different climatic zones. Microbiology knows them as "social amoeba" which describes their ability to form fruiting bodies, built up by a huge amount of up to 100.000 individuals. Dictyostelium disciodeum amoebae grow as separate, independent cells but interact to form multicellular structures when challenged by adverse conditions such as starvation. The individual cells signal each other low nutrient availability by releasing the chemo-attractant cAMP and aggregate by chemotaxis, forming a slug that is surrounded by an extracellular matrix and move away from a low nutrient location. Subsequent differentiation processes, depending on cell-to-cell communication, safeguard the organism to survive times of scarce nutritional resources.

Many of the molecular and cellular processes in complex organisms appear to have arisen in primitive precursor cells and have remained fundamentally unchanged throughout evolution. Basic processes of development such as differential cell sorting, pattern formation, stimulus-induced gene expression, and cell-type regulation are common to Dictyostelium which are described in a plethora of research papers focusing on the genetic regulation mechanisms for these specific functions.

The lifecycle of Dictyostelium disciodeum resolves into two basically different Phases. As long as nutrients (bacteria) are available the individuals exist as free living single cell amoebas, which crawl around and feed on bacteria. The different individual cells divide and grow but do not recognize each other. But as the nutrients get scarce, the cells begin to release the chemical signalling molecule cAMP which effects a messaging event. cAMP triggers a process that conducts the amoebas to start moving towards an aggregation centre. This centre is defined by a single cell, which emits periodic pulses of cAMP, which by this action affects also neighbouring cells to emit the signalling molecule cAMP. The cells now receive the signal, emit the signal by them self and start to move towards the origin of the received signal. The consequences of this attitude are wave-like cell movements towards the founder cell in the aggregation centre. Many thousands of individual organisms aggregate in this manner and start to build up a multi-cellular organism which in further differentiating steps builds specialized cell types (see fig.2). 


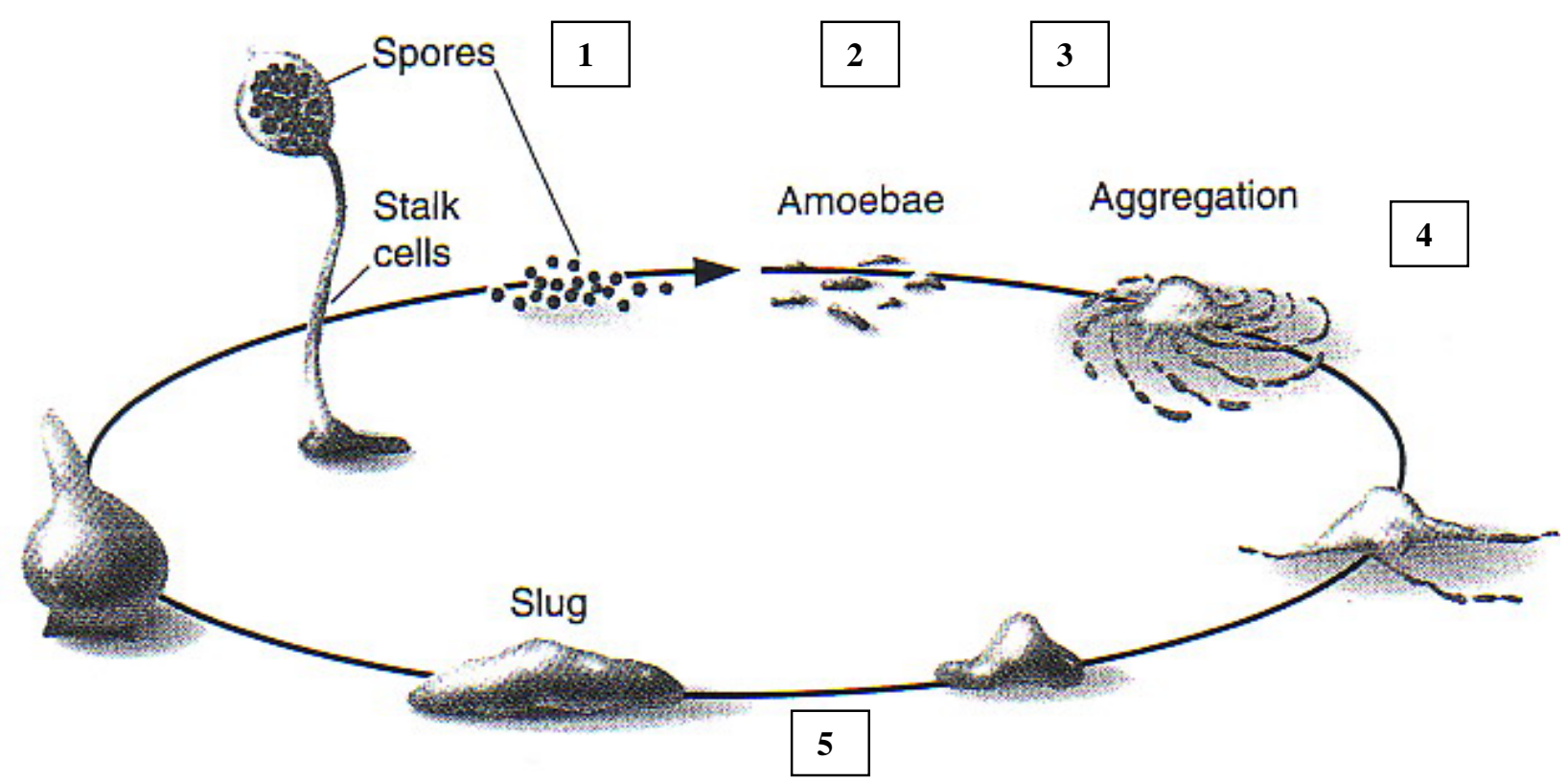

Fig. 2. The life cycle of Dictyostelium discoideum. Solitary amoebae prey on bacteria in the forest soil. When prey becomes scarce, the amoebae aggregate, first forming a mound of 1.000 to 100.000 cells. Then they form a motile slug which moves up through the leaf litter towards light. Finally the slug develops into a fruiting body, with a stalk formed of dead cells that hold up the remaining cells that sporulate, which can be dispersed by the wind or transported by animals to a better location.

In the informational or semiotic context we can describe these events during the life cycle of Dictyostelium discoideum as follows:

1) Normal life situation: The cell behaves in its "normal" way, under "normal environmental" conditions. The free available energy $\Delta G^{\circ}$ ' is the driving force which feeds on an energetic level the metabolism of the individual cell and enables anabolic metabolism during growth and reproduction of the informational polymeric molecules during cell division.

2) Changing life situation: As the environmental conditions change, which is the case, when resources are getting scarce the cell starts to change its internal metabolic activities as a result of starvation. We consider this change of activity within the cell as a process of cognition, because it is a direct consequence generated by the cell as an event that takes place outside of the cell.

3) Reaction of changing life situation: As a direct result of this cognition event the cell starts to produce and release the signalling molecule CAMP. As mentioned above this molecular agent is the product of a specific enzymatic reaction as a result of inter-conversion of the ATP molecule. The signal molecule is released by a cell and recognised by the mediation of specific receptors of another cell. This cell by itself starts also to produce the signalling molecule and an avalanche-like phenomenon can be observed as described in many research papers over the past decade. Speaking in biosemiotic terms this event can be understood as a co-operation-directed form of communication.

4) Aggregation: The next event that occurs is the development of a super-subject out of the former cosubjects. This activity is a behaviour of the cell, that directly emerges as consequence of the molecular communication process and can be described as form of co-operation.

5) The collective behaviour of this new cellular super-subject known as slug leads to a differentiated organism with new structures (stalk cells and generative spores) and a new function which is in this case the survival under altered environmental conditions.

As described in this example with the relative simple organism Dictyostelium discoideum, we see here an event that can be analogized with events occurring in much more complex organisms and that can be described as an very early stage in evolution where the notions cognition, communication and cooperation already can be applied, if we try to look through the lenses of biosemiotics. 


\section{References}

Ellersdorfer, Günther/Hofkirchner, Wolfgang (1994) Informationsstrukturen auf zellulärer Ebene und Fragen des Reduktionismus/Antireduktionismus. In: Wessel, K.-F./Naumann, F. (Eds.) (1994) Kommunikation und Humanontogenese. Bielefeld. Kleine. pp. 105-115.

Fenzl, Norbert, et al. (1996) On the Genesis of Information Structures. A View that is neither Reductionist nor Holistic. In Kornwachs, K./Jacoby, K. (Eds.) (1996) Information, New Questions to a Multidisciplinary Concept. Berlin. Akademie Verlag. pp. 271-283.

Fenzl, Norbert/Hofkirchner, Wolfgang/Stockinger, Gottfried (Eds.) (1998) Information und Selbstorganisation. Annäherungen an eine vereinheitlichte Theorie der Information. Innsbruck. Studienverlag

Gibson, James J. (1950) The perception of the visual world. Boston. Houghton Mifflin.

Gibson, James J. (1966) The senses considered as perceptual systems. Boston. Houghton Mifflin

Gibson, James J. (1979) The ecological approach to visual perception. Boston. Houghton Mifflin.

Hofkirchner, Wolfgang, Stockinger, Gottfried (2003) Towards a Unified Theory of Information. In: 404nOtF0und, Vol. 1 (3), N. 24 (Jan.).

Hofkirchner, Wolfgang/Fuchs, Christian/Klauninger, Bert (2005) Informational Universe. A Praxeo-Onto-Epistemological Approach. In: Martikainen, Eeva (Ed.) (2005) Human Approaches to the Universe. Interdisciplinary Studies. Helsinki. Luther-Agricola-Seura. pp. 75-94.

Luhmann, Niklas (2001) Die Gesellschaft der Gesellschaft. 2 vols. Frankfurt am Main. Suhrkamp.

Maturana, Humbero R./Varela, Francisco J. (1992) The Tree of Knowledge. Boston. Shambala

Piaget, Jean (1976) Die Äquilibration der kognitiven Strukturen. Stuttgart: Klett-Cotta.

Piaget, Jean (1980). Abriß der genetischen Epistemologie. Stuttgart. Klett-Cotta.

Progogine, llya (1980). From being to becoming. San Francisco. Freeman.

Stryer, Lubert (1988) Biochemistry. 3rd Edition. New York. Freemann and Company. 